\title{
Radio star catalogue observed in San Juan (RSSJ95)
}

\author{
W.T. Manrique ${ }^{1}$, Lu. Lizhi $^{2}$, R. Perdomo ${ }^{3}$, R.C. Podesta ${ }^{1}$, and Wang Zezhi ${ }^{2}$ \\ 1 Observatorio Astronomico Felix Aguilar, Benavidez 8175, San Juan, Argentina \\ 2 Beijing Astronomical Observatory, Beijing 100080, China \\ 3 Observatorio Astronomico de La Plata, 1900 La Plata, Argentina
}

Received May 28; accepted August 31, 1998

\begin{abstract}
Using the data observed in San Juan with the photoelectric Astrolabe Mark II of the Beijing Astronomical Observatory from February, 1992 to March, 1997, the radio star catalogue in San Juan(RSSJ95) has been compiled. There are 69 radio stars in this catalogue. The positions of the radio stars are for the epoch of observation and the equinox J2000.0 and a system close to that of the system FK5. The mean precisions are $\pm 2.2 \mathrm{~ms}$ and $\pm 0.035^{\prime \prime}$ in right ascensions and declinations, respectively. The magnitudes of stars are from 0.9 to 10.7. The declinations are from -2.5 to $-60^{\circ}$. The mean epoch is 1995.1. Finally, the comparison results between the Hipparcos catalogue and RSSJ95 are given.
\end{abstract}

Key words: radio continuum: stars — astrometry reference system

\section{Introduction}

The photoelectric astrolabe Mark II (PA II) was made at Nanjing Astronomical Instrument Factory in 1974 and was put into operation since Mar. 1976 (Luo 1979). The aperture of object mirror is $200 \mathrm{~mm}$ and the equivalent focal length is $2400 \mathrm{~mm}$. The zenith distance observed is $30^{\circ}$.

Before modernization, the astrolabe was a semi-automatic instrument with a limiting magnitude of only 7.0. Since 1987, the astronomers of the Beijing Astronomical Observatory began to improve the instrument. Automatic observation with the instrument started in 1988. A new method of photon-counting was used for data processing in 1990. And the limiting magnitude is increased to 11.5.

Using the data observed with the instruments from 1976 to 1991, several general catalogues (Zhu et al. 1981;
Working Group of GCPA, 1983; Working Group of CGSC, 1991; Lu 1991) of stars had been compiled.

In 1992, the instrument was moved and installed at the San Juan Observatory (OAFA) in Argentina for observations of the catalogue of stars in the southern hemisphere.

Radio stars are suitable intermediaries for linking optical stellar reference frames to the quasi-inertial radio reference frame represented by compact extragalactic radio sources. From Feb. 1992 to Mar. 1997, we observed radio stars with the photoelectric astrolabe using the list provided by H.G. Walter at Astronomishes Rechen-Institut, Heidelbery (Walter 1990).

In 1996, we presented the preliminary results (Lu et al. 1996). In this paper we submit all results observed from Feb. 1992 to Mar. 1997. The positions of these radio stars are fixed for epoch of observation and the equinox J2000.0 and in system close to that of the FK5.

\section{The observation and reduction}

There are 384 stars which are from FK5/FK4 Supp. in 12 fundamental groups. The observations of radio stars were placed in the reference group of stars. The residuals of the radio stars are calculated applying the corrections of astronomical time, latitude and zenith distance found from fundamental group observations. Then the mean values of the residuals of radio stars are calculated by weighted average.

Assuming $V_{\mathrm{e}}$ and $V_{\mathrm{w}}$ are the residuals reduced to the mean instrumental system after adding the group corrections of reference groups at both eastern and western transits, the position corrections of the radio stars are determined in right ascension and declination from double transits by the formulas:

$\Delta \alpha=\frac{V_{\mathrm{e}}-V_{\mathrm{w}}}{30 \cos \varphi_{0}|\sin A|}$

and

$\Delta \delta=-\frac{V_{\mathrm{e}}+V_{\mathrm{w}}-2 K}{2 \cos q}$, 
where

$\varphi_{0}-$ the adopted value of latitude at the site of the instrument;

$A$ - the azimuth of a star observed, measured eastwards from north;

$q$ - the parallactic angle of a star as it transits the almucantar of the astrolabe.

We used the stars with $|\cos q|<0.2$ to calculate $2 K$ by averaging

$K=\frac{1}{2}\left(V_{\mathrm{e}}+V_{\mathrm{w}}\right)$

and obtain $2 K=0.018^{\prime \prime} \pm 0.003^{\prime \prime}$.

\section{Results}

From Feb. 1992 to Mar. 1997, there are 69 radio stars to be observed with the photoelectric astrolabe. Using the data, the radio star catalogue observed in San Juan (RSSJ95) has been compiled from double transits of given radio stars at both eastern and western passages. The mean number of observations of each star is about 132 (63 in the eastern passage, 69 in western passage). The mean precisions are $\pm 2.2 \mathrm{~ms}$ and $\pm 0.035^{\prime \prime}$ in right ascension and declination, respectively.

The Radio star catalogue observed in San Juan is given in Table 1. The description of each column is:

1: INCA: star number in the proposals of radio stars submitted to the Input Catalogue Consortium of Hipparcos;

2: HD: HD number;

3: SAO: SAO number;

4: Mag: visual magnitude;

5: Sp: spectral type;

6: $\alpha_{2000}$ : right ascension referred to equinox J2000.0 at observation epoch;

7: $\sigma_{\alpha}$ : mean errors of position in right ascension in unit of $0.001^{\mathrm{s}}$;

8: $\delta_{2000}$ : declination referred to equinox J2000.0 at observation epoch;

9: $\sigma_{\delta}$ : mean errors of position in declination in unit of $0.01^{\prime \prime}$
10: $N_{\mathrm{e}}$ : number of observations in the eastern transit;

11: $N_{\mathrm{w}}$ : number of observations in the western transit;

12: Epoch: mean epoch of observations.

There are 68 radio stars commented RSSJ95 and the Hipparcos catalogue provided by CDS at nearly identical observational epochs. So we try to do a comparison between respective positions after reducing to the epoch of the Hipparcos catalogue, 1991.25, using the proper motions of the Hipparcos catalogue. The differences in the sense (Hipparcos - RSSJ95) are shown in Table 2.

In Table 2, the columns are as follows:

1: INCA: star number in the proposals of radio stars submitted to the Input Catalogue Consortium of Hipparcos;

2: HIP: number in Hipparcos catalogue;

3: $\alpha_{\text {hip }}$ : right ascension of Hipparcos catalogue referred to equinox J2000.0 at epoch 1991.25;

4: $\delta_{\text {hip }}$ : declination of Hipparcos catalogue referred to equinox J2000.0 at epoch 1991.25;

5: $\alpha_{\mathrm{rssj}}$ : right ascension of RSSJ95 referred to equinox J2000.0 at epoch 1991.25;

6: $\delta_{\text {rssj }}$ : declination of RSSJ95 referred to equinox J2000.0 at epoch 1991.25;

7: $\Delta \alpha$ : the difference in right ascension (HIP-RSSJ95) in unit of $0.001^{\mathrm{s}}$;

8: $\Delta \delta$ : the difference in declination (HIP-RSSJ95) in unit of $0.01^{\prime \prime}$.

\section{References}

Walter H.G., et al., 1990, A\&AS 86, 357

Lu Lizhi, 1991, Publ. Beijing Astron. Obs. 17, 1

Luo Dingjiang, et al., 1979, Publ. Beijing Astron. Obs. 1, 56

Working Group of GCPA, 1983, Acta Astron. Sin. 24, 267

Working Group of CGSC, 1991, Publ. House of Surveying and Mapping, Beijing

Lu Lizhi, Luo Dingjiang, et al., 1980, Acta Astron. Sin. 22, 305

Zhu Yuanxian, Lu Lizhi, et al., 1981, Publ. Beijing Astron. Obs. 1,1

Lu Lizhi, Manrique W.T., Perdomo R., 1996, A\&AS 118, 323 
Table 1. Radio star catalogue observed in San Juan

\begin{tabular}{|c|c|c|c|c|c|c|c|c|c|c|c|}
\hline \multirow[t]{2}{*}{ INCA } & \multirow[t]{2}{*}{$\mathrm{HD}$} & \multirow[t]{2}{*}{$\mathrm{SAO}$} & \multirow[t]{2}{*}{ Mag } & \multirow[t]{2}{*}{$\mathrm{Sp}$} & $\alpha_{2000}$ & \multirow{2}{*}{$\begin{array}{c}\sigma_{\alpha} \\
\mathrm{ms}\end{array}$} & $\delta_{2000}$ & \multirow{2}{*}{$\begin{array}{c}\sigma_{\delta} \\
0.01^{\prime \prime}\end{array}$} & \multirow[t]{2}{*}{$N_{\mathrm{e}}$} & \multirow[t]{2}{*}{$N_{\mathrm{w}}$} & \multirow{2}{*}{$\begin{array}{l}\text { Epoch } \\
1900+\end{array}$} \\
\hline & & & & & $\mathrm{h} \quad \mathrm{m} \quad \mathrm{s}$ & & & & & & \\
\hline 2003 & 1835 & 147237 & 6.39 & G2 & 02251.661 & 1.7 & -121234.26 & 2.5 & 68 & 83 & 95.37 \\
\hline 2701 & 6882 & 232306 & 3.91 & F7 & 10823.074 & 2.6 & -551444.96 & 2.5 & 69 & 56 & 95.49 \\
\hline 1010 & 7672 & 129204 & 5.35 & G5 & 11636.319 & 5.6 & -23001.18 & 2.1 & 51 & 42 & 95.40 \\
\hline 2652 & 10909 & 67287 & 8.10 & G5 & 14641.592 & 1.6 & -240050.69 & 5.4 & 53 & 45 & 94.94 \\
\hline 2653 & 17084 & 193879 & 8.10 & G5 & 24325.544 & 1.4 & & & 110 & 75 & 94.95 \\
\hline 2703 & 19754 & 130323 & 7.80 & K0 & 31038.501 & 2.4 & -52338.08 & 1.9 & 51 & 92 & 95.83 \\
\hline 2602 & 21985 & 130554 & 8.29 & A4 & 33225.155 & 5.0 & -31847.90 & 2.5 & 33 & 90 & 95.46 \\
\hline 1031 & 25038 & 216568 & 6.90 & $\mathrm{~K} 2$ & 35701.776 & 1.4 & & & 65 & 106 & 95.18 \\
\hline 1032 & 26354 & 233401 & 9.30 & K1 & 40729.129 & 2.2 & -523416.14 & 3.0 & 44 & 102 & 95.49 \\
\hline 2083 & 26337 & 130994 & 7.10 & G2 & 40940.889 & 2.0 & -75334.83 & 2.1 & 52 & 52 & 94.81 \\
\hline 1043 & 30050 & 149847 & 8.40 & A & 44345.829 & 2.3 & -104056.12 & 3.2 & 53 & 27 & 95.48 \\
\hline 2657 & 34198 & 170320 & 7.10 & K0 & 51430.552 & 1.1 & -261231.14 & 4.3 & 111 & 106 & 95.72 \\
\hline 2115 & 37017 & 132317 & 6.54 & B2 & 53521.887 & 4.8 & -42939.07 & 3.2 & 25 & 29 & 94.16 \\
\hline 1060 & 37847 & 170678 & 7.00 & G3 & 54039.703 & 1.6 & -201755.79 & 4.1 & 46 & 42 & 94.58 \\
\hline 1061 & 37806 & 132452 & 8.00 & A0 & 54102.307 & 5.1 & -24300.87 & 2.1 & 31 & 38 & 95.43 \\
\hline 2704 & 39576 & 170952 & 9.50 & G0 & 55215.990 & 2.2 & & & 29 & 29 & 94.58 \\
\hline 1062 & 39937 & 234181 & 7.30 & F7 & 55220.196 & 3.5 & -570921.94 & 2.4 & 45 & 70 & 95.15 \\
\hline 1076 & 51268 & 234770 & 9.10 & $\mathrm{~K} 2$ & 65333.583 & 2.2 & -545258.96 & 2.2 & 63 & 34 & 95.22 \\
\hline 1075 & 50896 & 172546 & 6.71 & WN & 65413.051 & 1.4 & -235541.97 & 4.9 & 41 & 83 & 95.03 \\
\hline 1078 & 54791 & 218478 & 9.90 & G8 & 70821.047 & 1.5 & & & 81 & 48 & 95.55 \\
\hline 1079 & 56096 & 218549 & 2.60 & M5 & 71332.269 & 1.7 & & & 116 & 46 & 95.25 \\
\hline 2603 & 57167 & 152724 & 5.70 & $\mathrm{~F} 1$ & 71928.123 & 1.6 & -162342.24 & 3.2 & 36 & 114 & 94.52 \\
\hline 1083 & 60414 & 153072 & 4.90 & M2 & 73347.969 & 1.7 & -143126.04 & 3.0 & 45 & 55 & 95.14 \\
\hline 2659 & 61245 & 218831 & 7.00 & K0 & 73613.790 & 2.0 & & & 38 & 100 & 94.83 \\
\hline 1087 & 66811 & 198752 & 2.25 & $\mathrm{O} 5$ & 80335.066 & 2.0 & & & 44 & 44 & 94.82 \\
\hline 2660 & 71071 & 135893 & 7.80 & G5 & 82514.124 & 2.7 & -71012.90 & 2.6 & 38 & 44 & 94.65 \\
\hline 2661 & 72688 & 199353 & 6.36 & K0 & 83258.524 & 2.0 & & & 30 & 78 & 95.34 \\
\hline 1092 & 77137 & 176805 & 7.20 & G2 & 85942.745 & 1.4 & & & 79 & 46 & 95.64 \\
\hline 1093 & 81410 & 177412 & 7.45 & K0 & 92449.031 & 1.2 & -234934.75 & 4.2 & 59 & 112 & 95.34 \\
\hline 2707 & 82558 & 155272 & 7.50 & K0 & 93225.658 & 2.1 & -111104.78 & 3.0 & 35 & 46 & 95.14 \\
\hline 1097 & 83442 & 221347 & 8.84 & K2 & 93712.991 & 1.7 & & & 48 & 78 & 95.14 \\
\hline 2663 & 94389 & 201857 & 8.20 & $\mathrm{~K} 2$ & 105314.885 & 1.2 & & & 91 & 95 & 95.49 \\
\hline 1112 & 96751 & 0 & 9.80 & G3 & 110756.348 & 1.9 & -510708.06 & 3.2 & 37 & 98 & 95.41 \\
\hline 1114 & 98803 & 222744 & 9.00 & G6 & 112144.513 & 1.9 & -495407.79 & 3.7 & 59 & 70 & 95.22 \\
\hline 1116 & 101309 & 202671 & 8.10 & G5 & 113922.237 & 1.6 & & & 98 & 38 & 94.09 \\
\hline 1119 & 102077 & 222993 & 8.90 & K0 & 114438.547 & 1.6 & -492502.38 & 3.5 & 97 & 114 & 94.71 \\
\hline 1123 & 103197 & 0 & 9.90 & K1 & 115253.007 & 2.6 & -501734.07 & 5.0 & 84 & 43 & 95.19 \\
\hline 1124 & 103855 & 223131 & 9.20 & G8 & 115726.717 & 2.0 & -483930.85 & 4.7 & 100 & 48 & 95.19 \\
\hline 2605 & 105452 & 180505 & 4.02 & F2 & 120824.788 & 1.5 & -244343.88 & 5.5 & 99 & 33 & 95.50 \\
\hline 2664 & 106225 & 138652 & 8.30 & K0 & 121320.701 & 2.3 & -90447.02 & 2.7 & 54 & 49 & 94.79 \\
\hline 2607 & 115122 & 157818 & 8.98 & A2 & 131520.756 & 2.2 & -172816.97 & 4.7 & 17 & 111 & 94.11 \\
\hline 1141 & 117600 & 224225 & 9.90 & $\mathrm{~K} 2$ & 133216.205 & 2.9 & -472506.47 & 8.0 & 23 & 151 & 94.62 \\
\hline 2665 & 118238 & 204640 & 8.84 & $\mathrm{~K} 2$ & 133608.303 & 1.5 & & & 92 & 56 & 95.68 \\
\hline 2709 & 123485 & 224722 & 8.90 & A0 & 140902.201 & 2.2 & & & 93 & 37 & 95.13 \\
\hline 1154 & 127535 & 252794 & 9.70 & $\mathrm{~K} 1$ & 143416.110 & 8.8 & -602428.65 & 2.5 & 48 & 14 & 95.45 \\
\hline 1155 & 128171 & 158665 & 9.80 & G5 & 143548.434 & 1.7 & -180211.18 & 3.8 & 40 & 97 & 95.30 \\
\hline 2609 & 132742 & 140270 & 4.91 & A0 & 150058.380 & 1.9 & $\begin{array}{lll}-8 & 31 & 08.23\end{array}$ & 2.2 & 49 & 71 & 94.38 \\
\hline 2290 & 136905 & 140499 & 7.40 & K0 & 152326.070 & 2.2 & -63637.19 & 2.0 & 77 & 85 & 95.37 \\
\hline 1168 & 139084 & 242791 & 8.10 & G5 & 153857.586 & 4.3 & -574226.80 & 2.8 & 31 & 37 & 95.30 \\
\hline 2610 & 142217 & 207103 & 8.20 & F5 & 155427.052 & 1.1 & & & 129 & 120 & 95.30 \\
\hline
\end{tabular}


Table 1. continued

\begin{tabular}{|c|c|c|c|c|c|c|c|c|c|c|c|}
\hline \multirow[t]{2}{*}{ INCA } & \multirow[t]{2}{*}{ HD } & \multirow[t]{2}{*}{$\mathrm{SAO}$} & \multirow[t]{2}{*}{ Mag } & \multirow[t]{2}{*}{ Sp } & $\alpha_{2000}$ & \multirow{2}{*}{$\begin{array}{l}\sigma_{\alpha} \\
\mathrm{ms}\end{array}$} & $\delta_{2000}$ & \multirow{2}{*}{$\begin{array}{c}\sigma_{\delta} \\
0.01^{\prime \prime}\end{array}$} & \multirow[t]{2}{*}{$N_{\mathrm{e}}$} & \multirow[t]{2}{*}{$N_{\mathrm{w}}$} & \multirow{2}{*}{$\begin{array}{l}\text { Epoch } \\
1900+\end{array}$} \\
\hline & & & & & $\mathrm{h} \quad \mathrm{m} \quad \mathrm{s}$ & & & & & & \\
\hline 1173 & 146550 & 0 & 10.10 & F6 & 161841.508 & 2.1 & & & 122 & 26 & 94.80 \\
\hline 1176 & 148478 & 184415 & .90 & M1 & 162924.461 & 1.4 & & & 38 & 64 & 95.26 \\
\hline 2710 & 152556 & 141428 & 8.10 & K5 & 165417.932 & 2.2 & $\begin{array}{lll}-6 & 42 & 42.62\end{array}$ & 2.1 & 94 & 33 & 94.81 \\
\hline 2666 & 156026 & 185213 & 6.70 & $\mathrm{~K} 2$ & 171613.542 & 1.5 & & & 68 & 58 & 95.10 \\
\hline 2711 & 161741 & 209291 & 8.20 & B9 & 174847.620 & 1.7 & & & 76 & 34 & 94.53 \\
\hline 1190 & 164794 & 186204 & 5.97 & O5 & 180352.449 & 1.8 & -242138.72 & 6.2 & 55 & 42 & 94.60 \\
\hline 1191 & 165141 & 228707 & 8.00 & G8 & 180700.246 & 1.9 & $-48 \quad 1450.36$ & 4.8 & 45 & 62 & 93.99 \\
\hline 1198 & 169515 & 161458 & 9.70 & O9 & 182531.469 & 2.7 & -124124.39 & 4.3 & 30 & 44 & 95.30 \\
\hline 1202 & 174429 & 245781 & 8.36 & K0 & 185305.868 & 2.0 & $\begin{array}{llll}-50 & 10 & 49.51\end{array}$ & 3.9 & 58 & 85 & 95.17 \\
\hline 1208 & 181809 & 188043 & 6.90 & K0 & 192240.303 & 1.2 & -203834.01 & 3.3 & 98 & 119 & 95.26 \\
\hline 1209 & 181943 & 162546 & 9.20 & G5 & 192257.246 & 2.5 & -141531.77 & 4.3 & 31 & 71 & 94.54 \\
\hline 1210 & 182776 & 229695 & 9.60 & K0 & 192805.557 & 1.5 & & & 134 & 72 & 94.62 \\
\hline 1212 & 185510 & 143657 & 8.30 & G5 & 193938.810 & 2.0 & -60349.34 & 1.7 & 71 & 137 & 95.42 \\
\hline 2715 & 187949 & 163080 & 6.46 & A2 & 195306.389 & 1.5 & -143611.26 & 2.7 & 49 & 132 & 95.13 \\
\hline 1226 & 195040 & 189349 & 9.00 & K0 & 202936.871 & 2.2 & -210734.39 & 6.0 & 29 & 71 & 95.49 \\
\hline 1238 & 202134 & 212824 & 7.80 & K0 & 211452.733 & 2.2 & & & 25 & 56 & 94.88 \\
\hline 2470 & 205249 & 164484 & 8.00 & G5 & 213416.566 & 1.2 & -132901.50 & 1.9 & 153 & 138 & 95.21 \\
\hline 1242 & 206046 & 164558 & 10.77 & G5 & 213948.908 & 1.7 & -160021.10 & 3.4 & 44 & 93 & 95.48 \\
\hline 2476 & 207098 & 164644 & 2.81 & A7 & 214702.362 & 1.6 & -160736.81 & 3.1 & 96 & 71 & 94.98 \\
\hline
\end{tabular}

Table 2. The difference of positions (HIP-RSSJ95)

\begin{tabular}{|c|c|c|c|c|c|c|c|c|c|}
\hline \multirow[t]{2}{*}{ INCA } & \multirow[t]{2}{*}{ HIP } & \multicolumn{2}{|r|}{$\alpha_{\text {hip }}$} & $\delta_{\text {hip }}$ & \multicolumn{2}{|r|}{$\alpha_{\mathrm{rssj}}$} & $\delta_{\mathrm{rssj}}$ & \multirow{2}{*}{$\begin{array}{l}\Delta \alpha \\
\mathrm{ms}\end{array}$} & \multirow{2}{*}{$\begin{array}{c}\Delta \delta \\
0.01^{\prime \prime}\end{array}$} \\
\hline & & $\mathrm{h}$ & $\mathrm{m} \quad \mathrm{s}$ & & $\mathrm{h}$ & $\mathrm{m} \mathrm{s}$ & & & \\
\hline 2003 & 1803 & 022 & 51.5530 & $-12 \quad 1234.506$ & 022 & 51.5502 & $-12 \quad 1234.509$ & 2.8 & 0.3 \\
\hline 2701 & 5348 & 108 & 23.0601 & -551444.996 & 108 & 23.0635 & -551445.086 & -3.4 & 9.0 \\
\hline 1010 & 5951 & 116 & 36.3467 & -23000.766 & 116 & 36.3469 & -23000.915 & -0.2 & 14.9 \\
\hline 2652 & 8281 & 146 & 41.5509 & -240051.153 & 146 & 41.5513 & -240051.049 & -0.4 & -10.4 \\
\hline 2653 & 12716 & 243 & 25.5086 & -375541.893 & 243 & 25.5193 & & -10.7 & \\
\hline 2703 & 14763 & 310 & 38.4881 & -52338.100 & 310 & 38.4842 & -52338.095 & 3.9 & -0.5 \\
\hline 2602 & 16496 & 332 & 25.1439 & -31847.729 & 332 & 25.1635 & -31847.727 & -19.6 & -0.2 \\
\hline 1031 & 18482 & 357 & 01.7716 & -400213.071 & 357 & 01.7735 & & -1.9 & \\
\hline 1032 & 19248 & 407 & 29.0608 & -523415.189 & 407 & 29.0606 & -523415.200 & 0.2 & 1.1 \\
\hline 2083 & 19431 & 409 & 40.8728 & -75335.177 & 409 & 40.8807 & -75335.191 & -7.9 & 1.4 \\
\hline 1043 & 22000 & 443 & 45.8268 & -104056.100 & 443 & 45.8272 & -104056.161 & -0.4 & 6.1 \\
\hline 2657 & 24430 & 514 & 30.5313 & -261230.725 & 514 & 30.5333 & -261230.852 & -2.0 & 12.7 \\
\hline 2115 & 26233 & 535 & 21.8655 & -42939.029 & 535 & 21.8866 & -42939.073 & -21.1 & 4.4 \\
\hline 1060 & 26714 & 540 & 39.7010 & -201755.489 & 540 & 39.6968 & -201755.754 & 4.2 & 26.5 \\
\hline 1061 & 26752 & 541 & 02.2904 & -24300.722 & 541 & 02.3059 & -24300.867 & -15.5 & 14.5 \\
\hline 2704 & 27727 & 552 & 15.9839 & -283924.748 & 552 & 15.9886 & & -4.7 & \\
\hline 1062 & 27737 & 552 & 20.1821 & -570921.600 & 552 & 20.1874 & -570921.633 & -5.3 & 3.3 \\
\hline 1076 & 33093 & 653 & 33.5566 & -545259.299 & 653 & 33.5606 & -545259.331 & -4.0 & 3.2 \\
\hline 1075 & 33165 & 654 & 13.0465 & -235542.053 & 654 & 13.0521 & -235541.988 & -5.6 & -6.5 \\
\hline 1078 & 34436 & 708 & 21.0406 & -415438.319 & 708 & 21.0419 & & -1.3 & \\
\hline
\end{tabular}


Table 2. continued

\begin{tabular}{|c|c|c|c|c|c|c|c|}
\hline \multirow[t]{2}{*}{ INCA } & \multirow[t]{2}{*}{ HIP } & $\alpha_{\text {hip }}$ & $\delta_{\text {hip }}$ & $\alpha_{\mathrm{rssj}}$ & $\delta_{\text {rssj }}$ & \multirow{2}{*}{$\begin{array}{l}\Delta \alpha \\
\mathrm{ms}\end{array}$} & \multirow{2}{*}{$\begin{array}{c}\Delta \delta \\
0.01^{\prime \prime}\end{array}$} \\
\hline & & $\mathrm{h} \mathrm{m} \mathrm{s}$ & 0,11 & $\mathrm{~m} \mathrm{~s}$ & & & \\
\hline 1079 & 34922 & 71332.2309 & -443825.906 & 71332.2289 & & 2.0 & \\
\hline 2603 & 35487 & 71928.0814 & -162341.686 & 71928.0854 & -162341.794 & -4.0 & 10.8 \\
\hline 1083 & 36773 & 73347.9679 & -143126.044 & 73347.9711 & -143126.054 & -3.2 & 1.0 \\
\hline 2659 & 36992 & 73613.7799 & -445727.298 & 73613.7799 & & 0.0 & \\
\hline 1087 & 39429 & 80335.0701 & -400011.478 & 80335.0756 & & -5.5 & \\
\hline 2660 & 41274 & 82514.1507 & -71012.767 & 82514.1509 & -71012.867 & -0.2 & 10.0 \\
\hline 2661 & 41939 & 83258.5138 & -343802.567 & 83258.5301 & & -16.3 & \\
\hline 1092 & 44164 & 85942.7510 & -274858.301 & 85942.7596 & & -8.6 & \\
\hline 1093 & 46159 & 92449.0391 & -234934.434 & 92449.0422 & -234934.618 & -3.1 & 18.4 \\
\hline 2707 & 46816 & 93225.7159 & -111104.992 & 93225.7236 & -111104.916 & -7.7 & -7.6 \\
\hline 1097 & 47206 & 93713.0182 & -420114.654 & 93713.0163 & & 1.9 & \\
\hline 2663 & 53227 & 105314.8848 & -325918.994 & 105314.8927 & & -7.9 & \\
\hline 1112 & 54401 & 110756.3925 & -510707.958 & 110756.4060 & -510708.204 & -13.5 & 24.6 \\
\hline 1114 & 55478 & 112144.5184 & -495407.700 & 112144.5215 & -495407.781 & -3.1 & 8.1 \\
\hline 1116 & 56851 & 113922.2374 & -392307.143 & 113922.2374 & & 0.0 & \\
\hline 1119 & 57269 & 114438.5864 & -492502.351 & 114438.5957 & -492502.215 & -9.3 & -13.6 \\
\hline 1123 & 57931 & 115253.0522 & -501734.225 & 115253.0401 & -501734.099 & 12.1 & -12.6 \\
\hline 1124 & 58310 & 115726.7308 & -483930.752 & 115726.7307 & -483930.849 & 0.1 & 9.7 \\
\hline 2605 & 59199 & 120824.7527 & -244343.608 & 120824.7567 & -244343.713 & -4.0 & 10.5 \\
\hline 2664 & 59600 & 121320.7009 & -90446.877 & 121320.7038 & -90447.018 & -2.9 & 14.1 \\
\hline 2607 & 64672 & 131520.7662 & -172816.740 & 131520.7660 & -172816.907 & 0.2 & 16.7 \\
\hline 1141 & 66032 & 133216.2427 & -472506.080 & 133216.2537 & -472506.212 & -11.0 & 13.2 \\
\hline 2665 & 66358 & 133608.3219 & -332844.771 & 133608.3037 & & 18.2 & \\
\hline 2709 & 69130 & 140902.2093 & -453430.575 & 140902.2054 & & 3.9 & \\
\hline 1155 & 71380 & 143548.4310 & -180211.373 & 143548.4398 & -180211.104 & -8.8 & -26.9 \\
\hline 2609 & 73473 & 150058.3877 & -83108.166 & 150058.3940 & -83108.219 & -6.3 & 5.3 \\
\hline 2290 & 75325 & 152326.0581 & -63636.683 & 152326.0699 & -63636.682 & -11.8 & -0.1 \\
\hline 1168 & 76629 & 153857.6023 & -574226.411 & 153857.6127 & -574226.371 & -10.4 & -4.0 \\
\hline 2610 & 77896 & 155427.0549 & -302531.054 & 155427.0577 & & -2.8 & \\
\hline 1173 & 79913 & 161841.5107 & -424229.729 & 161841.5147 & & -4.0 & \\
\hline 1176 & 80763 & 162924.4675 & -262555.006 & 162924.4640 & & 3.5 & \\
\hline 2710 & 82699 & 165417.9303 & -64242.438 & 165417.9326 & -64242.575 & -2.3 & 13.7 \\
\hline 2666 & 84478 & 171613.6754 & -263236.299 & 171613.6796 & & -4.2 & \\
\hline 2711 & 87191 & 174847.5985 & -350325.626 & 174847.6184 & & -19.9 & \\
\hline 1190 & 88469 & 180352.4445 & -242138.613 & 180352.4488 & -242138.714 & -4.3 & 10.1 \\
\hline 1191 & 88743 & 180700.2464 & -481450.304 & 180700.2450 & -481450.384 & 1.4 & 8.0 \\
\hline 1198 & 90303 & 182531.4774 & -124124.177 & 182531.4685 & -124124.385 & 8.9 & 20.8 \\
\hline 1202 & 92680 & 185305.8592 & -501049.148 & 185305.8612 & -501049.182 & -2.0 & 3.4 \\
\hline 1208 & 95244 & 192240.2969 & -203833.558 & 192240.3015 & -203833.589 & -4.6 & 3.1 \\
\hline 1209 & 95266 & 192257.2314 & -141531.960 & 192257.2341 & -141531.748 & -2.7 & -21.2 \\
\hline 1210 & 95714 & 192805.5466 & -405004.835 & 192805.5487 & & -2.1 & \\
\hline 1212 & 96714 & 193938.8041 & -60349.218 & 193938.8036 & -60349.224 & 0.5 & 0.6 \\
\hline 2715 & 97849 & 195306.3859 & -143611.089 & 195306.3892 & -143611.064 & -3.3 & -2.5 \\
\hline 1226 & 101098 & 202936.8542 & -210734.640 & 202936.8704 & -210734.349 & -16.2 & -29.1 \\
\hline 1238 & 104894 & 211452.7391 & $-31 \quad 1100.813$ & 211452.7484 & & -9.3 & \\
\hline 2470 & 106497 & 213416.5606 & -132901.516 & 213416.5617 & -132901.514 & -1.1 & -0.2 \\
\hline 1242 & 106961 & 213948.8892 & -160021.016 & 213948.8950 & -160021.091 & -5.8 & 7.5 \\
\hline 2476 & 107556 & 214702.2853 & -160735.637 & $\begin{array}{lll}21 & 47 & 02.2939\end{array}$ & -160735.705 & -8.6 & 6.8 \\
\hline
\end{tabular}

